\section{Cestode Zoonoses: Echinococcosis and Cysticercosis: An Emergent and Global Problem}

\author{
Philip Craig and \\ Zbigniew Pawlowski, editors \\ Vol. 341 NATO Science Series, \\ IOS Press, Amsterdam \\ 410 pages, hardcover \\ ISBN: 1-58603-220-8
}

Price: US $\$ 100$

This book is a collection of short articles written by the participants of a research workshop held in Poznan, Poland, in September 2000. The workshop, supported by NATO Scientific Affairs, focused on the three major larval cestode diseases of humans: Taenia solium neurocysticercosis, Echinococcus granulosus cystic hydatidosis, and E. multilocularis alveolar hydatidosis. The format and depth of the articles are variable, but readers familiar with these parasites will find the book to be a convenient collection of new information on the subject. A shortcoming is that the book's preface and summary are each limited to a single page.

Perhaps most interesting for readers of the Emerging Infectious Diseases Journal are the reviews of epidemiologic data related to the emergence or reemergence of these three diseases. In sub-Saharan Africa, for example, neurocysticercosis has emerged as being more widely distributed than previously assumed and is a major cause of epilepsy. Surgery for pediatric cystic echinococcosis in Kyrgystan increased threefold during the period 1993-1998 (reaching 6 cases/100,000), suggesting new transmission probably related to worsening economic conditions after the collapse of the former Soviet Union. Surveillance for human cases of alveolar echinococcosis (which can have a mortality rate of $90 \%$ if untreated) is being strengthened in western Europe, given that E. multilocularis infection rates in foxes have increased in recent years. The book contains other valuable updates on diagnostics, immunology and vaccines, imaging and clinical management, geographic information systems and ecology, veterinary medicine, and community-based control programs. Readers with an interest in helminthology will find this book most useful.

Frank O. Richards, Jr.

The Carter Center, Atlanta, Georgia, USA

Address for correspondence: Frank O. Richards, Jr., The Carter Center, 453 Freedom Parkway, Atlanta, GA 30307, USA; fax: 770-420-5100; e-mail: sdsulli@emory.edu

\section{Immunology of Infectious Diseases}

\section{By Stefan H.E. Kaufmann, Alan Sher, and Rafi Ahmed}

\section{American Society for \\ Microbiology Press \\ Washington, D.C. \\ 520 pages}

ISBN: 1-55581-214-7

Price: US $\$ 115.95$

Whether an infectious disease agent is an "old acquaintance" or a new, emerging threat, the immune system's battle against it is usually the first line of defense it encounters. With vaccines and effective treatments often unavailable, the immune system's efforts to eradicate infectious agents or infected cells are frequently the only means to combat them. Understanding the immune systemas well as the infectious agent's tactics to undermine it - is of vital importance to the researcher and clinician. This textbook attempts to provide just this information.

Immunology of Infectious Diseases is a textbook in the best sense of the word, presenting its contents in a clear, structured manner. Instead of encylopedic coverage of every infectious disease agent known, a set of paradigmatic infections were selected on the basis of the depth of available knowledge. The book is divided into eight sections, each of which addresses a particular aspect of the host-infectious agent interaction, describing it in separate chapters for bacteria, fungi, parasitic eukaryotes, and viruses. So instead of discussing all aspects of viral diseases, the reader learns about the innate immune response to the various pathogens, chapter by chapter, in the respective section. Emphasis is thereby placed on the immune system's "point of view" about an infectious process, rather than on the microbe's.

After an introduction to the various classes of infectious disease agents, the book describes the immune responses directed against the different types of infections, proceeding from the innate to the acquired (adaptive). Discussion of the pathology of infections not eradicated by the immune system early on and the cunning strategies of the infectious microbes to evade immune attacks is followed by sections on immunogenetics and exploration of the immune system's interventions against two high-incidence infections, tuberculosis and AIDS.

Although the infections discussed in this book are not emerging ones in the strictest sense, the example of AIDS shows just how fast an infectious disease that was emerging, seemingly restricted to a subset of the population only two decades ago, can grow into a pandemic in a highly mobile, dense population at the end of the 20th century. Even tuberculosis, the "wasting disease" dreaded by our grandfathers' generation, which scientists believed to be under control, can be regarded as an emerging disease: Mycobacterium tuberculosis has stepped into the limelight again in the wake of HIV, which renders a growing number of people immunocompromised. 